\title{
Velocity Dependence from Resonant Self-Interacting Dark Matter
}

\author{
Xiaoyong Chu, ${ }^{1, *}$ Camilo Garcia-Cely, ${ }^{2, \dagger}$ and Hitoshi Murayama ${ }^{3,4,5,2, \$}$ \\ ${ }^{1}$ Institute of High Energy Physics, Austrian Academy of Sciences, Nikolsdorfer Gasse 18, 1050 Vienna, Austria \\ ${ }^{2}$ Deutsches Elektronen-Synchrotron DESY, Notkestrasse 85, 22607 Hamburg, Germany \\ ${ }^{3}$ Department of Physics, University of California, Berkeley, California 94720, USA \\ ${ }^{4}$ Kavli Institute for the Physics and Mathematics of the Universe (WPI), University of Tokyo, Kashiwa 277-8583, Japan \\ ${ }^{5}$ Ernest Orlando Lawrence Berkeley National Laboratory, Berkeley, California 94720, USA
}

(Received 23 October 2018; revised manuscript received 24 November 2018; published 22 February 2019)

\begin{abstract}
The dark matter density distribution in small-scale astrophysical objects may indicate that dark matter is self-interacting, while observations from clusters of galaxies suggest that the corresponding cross section depends on the velocity. Using a model-independent approach, we show that resonant self-interacting dark matter can naturally explain such a behavior. In contrast to what is often assumed, this does not require a light mediator. We present explicit realizations of this mechanism and discuss the corresponding astrophysical constraints.
\end{abstract}

DOI: 10.1103/PhysRevLett.122.071103

Dark matter (DM) makes up more than $80 \%$ of the matter in the Universe today and played a crucial role in forming stars, galaxies, and hence us. Yet its nature is unknown. Currently, the best pieces of information come from astrophysical observations. $N$-body simulations of collisionless DM predict astrophysical halos with DM density following a universal profile that scales as $\rho \propto r^{-3}$ in its outskirts but exhibits a central cusp, $\rho \propto r^{-\beta}$, with $\beta \simeq 1$, referred to as the Navarro-Frenk-White (NFW) profile [1-3]. Nevertheless, many studies show hints of a DM mass deficit in the inner regions of certain halos. Notably, observations indicate that numerous dwarf galaxies [4-6] and some low-surfacebrightness spiral galaxies [7-9] have a shallower central DM density, better described by a core of constant density, i.e., by $\beta \simeq 0$. This is known as the core-vs-cusp problem. Although it is more pressing in small-scale objects, shallower DM density profiles-with a slope of $\beta \simeq 0.5$ - have been reported for certain galaxy clusters $[10,11]$. Moreover, the DM mass deficit also manifests itself in halos that are less dense than what simulations suggest if they host the galaxies that we observe. This is the too-big-to-fail problem, observed for the subhalos of the Milky Way [12], Andromeda [13], and the Local Group [14].

Several explanations for these discrepancies have been discussed in the literature. The systematic uncertainties introduced in deriving DM distributions from observations of luminous objects are one of them. Most importantly, the

Published by the American Physical Society under the terms of the Creative Commons Attribution 4.0 International license. Further distribution of this work must maintain attribution to the author(s) and the published article's title, journal citation, and DOI. Funded by SCOAP ${ }^{3}$. motions of HI gas and stars may not be faithful tracers of the DM circular velocity [15-28]. Baryonic processes are another conceivable explanation for the discrepancies, since the aforementioned simulations include only collisionless DM. Solutions along this line include supernova-driven baryonic winds [29-32], DM heating due to star formation [33], and infalling baryonic clumps [34-37] as well as active galactic nuclei or black holes [38]. Nonetheless, there is no consensus on why systematic uncertainties or baryonic processes lead to a seemingly universal mass deficit at various scales.

A more exciting possibility consists of considering DM collisions in the inner regions of astrophysical objects [39]. This is known as self-interacting dark matter (SIDM). $N$-body simulations [40-45] confirm that DM scattering processes indeed reduce the central density of DM halos, providing a solution to both problems [46]. For a recent review, see [51].

The observed mass deficit is more appreciable in smallscale halos, where the DM velocity dispersion is relatively low. Therefore, a self-scattering cross section that decreases with the DM velocity can better fit observations [52], although a constant cross section is certainly not excluded due to the large uncertainties mentioned above. A longrange force induced by a light boson interacting with DM is often invoked to obtain a velocity-dependent cross section [39,53]. Other possibilities that do not involve a light mediator include exothermic inelastic scatterings [54,55] and self-heating DM [56-58].

The essence of this work is to discuss the resonant selfinteraction of DM (RSIDM) as another mechanism for achieving the desired velocity dependence of SIDM. Such a resonant behavior was first discussed for DM annihilation in Refs. [59-69] and applied to DM self-scattering in 

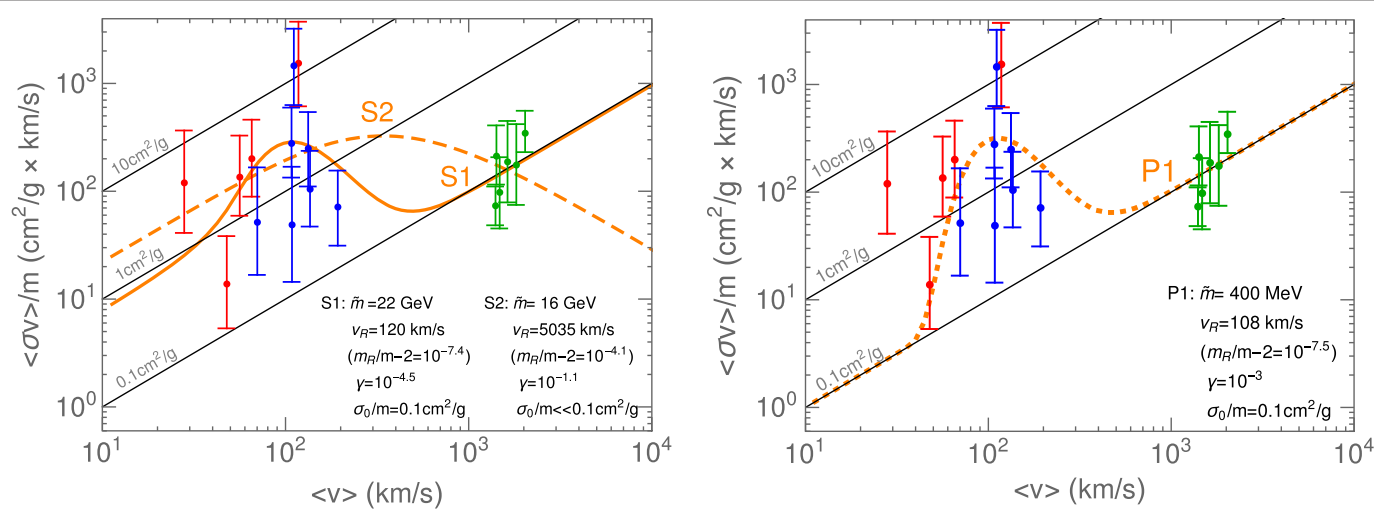

FIG. 1. RSIDM cross section per unit of mass as a function of the velocity. Best-fit curves to the data [52] for $S$-wave (left) and $P$-wave scatterings (right). The latter is also the best-fit curve for $L>1$ after rescaling the mass with Eq. (9). Here $\tilde{m}=m S^{-1 / 3}$. See the text for details.

specific scenarios [70-73]. Nevertheless, the velocity dependence of resonant self-scattering and its general astrophysical consequences have not been explored in detail. In this Letter, we do so in a model-independent way and show that resonant scattering is able to address the observed DM mass deficit at all astrophysical scales. Concrete DM scenarios and indirect searches are discussed later.

Resonant scattering in DM halos.-Numerous studies claim that the density distribution of certain DM halos does not follow a NFW profile in the inner region. In the SIDM hypothesis, this is due to DM collisions that thermalize the DM particles in such a region, thereby reducing its average density [39]. Hence, the inner profile is closely related to the velocity-averaged scattering cross section per unit of DM mass, $\langle\sigma v\rangle / m$, where [74]

$$
\langle\sigma v\rangle=\int_{0}^{v_{\max }} f\left(v, v_{0}\right) \sigma v d v, \quad f\left(v, v_{0}\right)=\frac{4 v^{2} e^{-v^{2} / v_{0}^{2}}}{\sqrt{\pi} v_{0}^{3}} .
$$

Here, $v$ is the relative velocity, which we assume to follow a Maxwell-Boltzmann distribution truncated at the escape velocity $v_{\max }$ of the corresponding halo. $v_{0}$ is a parameter related to the average relative velocity via $\langle v\rangle \simeq 2 v_{0} / \sqrt{\pi}$. Notice that in dwarf galaxies $\langle v\rangle \sim 20 \mathrm{~km} / \mathrm{s}$, whereas in clusters of galaxies $\langle v\rangle \sim 2000 \mathrm{~km} / \mathrm{s}$.

A semianalytical method has been proposed in Ref. [52] to infer the value of $\langle\sigma v\rangle / m$ for a given DM halo from observational data. The method was applied to five clusters from Ref. [11], seven low-surface-brightness spiral galaxies in Ref. [75], and six dwarf galaxies of The HI Nearby Galaxy Survey sample [76] (also see [77,78]). Figure 1 shows their results in green, blue, and red, respectively. The values presented here are for illustrative purpose and should be taken with caution due to the large uncertainties in extracting the cross sections from kinematical data. See, e.g., [79] for a recent study. Nonetheless, at face value, the figure demonstrates that a cross section independent of the velocity - the ones corresponding to the diagonal linescan hardly accommodate all points. Notice that the values of $\sigma / \mathrm{m}$ at cluster scales are in agreement with observations from the Bullet Cluster giving $\sigma / m \lesssim 1.3 \mathrm{~cm}^{2} / \mathrm{g}[80,81]$, which is one of the strongest constraints on DM selfinteractions.

Barring the uncertainties, the figure suggests that the cross section depends on $\langle v\rangle$. In this Letter, we propose that this is due to RSIDM. This takes place when there exists an intermediate particle, denoted as $R$, so that the total self-scattering cross section can be cast as a sum of a constant piece, $\sigma_{0}$, plus a Breit-Wigner resonance [82]. More explicitly, for nonrelativistic DM,

$$
\sigma=\sigma_{0}+\frac{4 \pi S}{m E(v)} \frac{\Gamma(v)^{2} / 4}{\left[E(v)-E\left(v_{R}\right)\right]^{2}+\Gamma(v)^{2} / 4},
$$

where the total kinetic energy and symmetry factor read, respectively,

$$
E(v)=\frac{1}{2} \frac{m}{2} v^{2} \quad \text { and } \quad S=\frac{2 J_{R}+1}{\left(2 J_{\mathrm{DM}}+1\right)^{2}} .
$$

Here, $J_{R}$ and $J_{\mathrm{DM}}$ are the spins of the resonance and the DM particles, respectively. $m / 2$ is the reduced mass. If DM has internal degrees of freedom other than its spin, they must be accounted for in $S$. The collision hits the resonance when $v=v_{R}$ and, hence, $E\left(v_{R}\right)=m_{R}-2 m$.

In addition, the width in Eq. (2) can be calculated in terms of the resonance self-energy by means of $\Gamma(v)=$ $\operatorname{Im} \Sigma(v) / m_{R}$. This, as well as the denominator in Eq. (2), assumes that the total width is dominated by the process $R \rightarrow$ DM DM. Besides that, Eq. (2) is completely general, as it directly follows from unitarity considerations of the scattering matrix [83]. In perturbative theories, the running width can be written as 


$$
\Gamma(v)=m_{R} \gamma v^{2 L+1} .
$$

Here, $L$ is the orbital angular momentum, $\Gamma\left(v_{R}\right)$ is the decay rate, and a constant $\gamma \lesssim O(1)$ characterizes the coupling between the resonance and DM. The factor $v^{2 L+1}$ accounts for the phase space and possible angular momentum suppression. Then we find $\langle\sigma v\rangle=\sigma_{0}\langle v\rangle+$ $256 \pi S \mathcal{I}_{L}\left(\gamma, v_{R}, v_{0}\right) / \mathrm{m}^{2}$, where a dimensionless

$$
\mathcal{I}_{L}\left(\gamma, v_{R}, v_{0}\right) \equiv \int_{0}^{v_{\max }} \frac{\gamma^{2} f\left(v, v_{0}\right) v^{4 L+1} d v}{\left(v^{2}-v_{R}^{2}\right)^{2}+16 \gamma^{2} v^{2(2 L+1)}}
$$

determines the nontrivial velocity dependence of the resonant self-scattering. For $S$-wave and $P$-wave scatterings, we calculate the best-fit parameter sets $S 1, S 2$, and $P 1$ based on the inferred data from Ref. [52] and show them in Fig. 1 [84]. $\sigma_{0} / m$ is fitted with the other parameters for $S 1$ and $P 1$, while for $S 2$ a negligible $\sigma_{0} / m \ll 0.1 \mathrm{~cm}^{2} / \mathrm{g}$ is taken as a prior. They all lead to $\chi^{2} /$ d.o.f. $\simeq 2$, in contrast to $\chi^{2} /$ d.o.f. $\simeq 6$ for the fit assuming only a constant cross section (we treat errors as uncorrelated). For $S 1$ and $P 1$, we show the 95\% C.L. contours in Fig. 2. Many comments are in order.

First, we have numerically checked that a precise knowledge of the escape velocity is not necessary for calculating $\mathcal{I}_{L}$. This is because Eq. (5) converges quite fast due to the Boltzmann factor. In fact, exact solutions exist in the limit $v_{\max } \gg v_{0}$, which will be implicitly applied hereafter for simplicity.

Second, to qualitatively understand Figs. 1 and 2, one can use the narrow-width approximation (NWA)
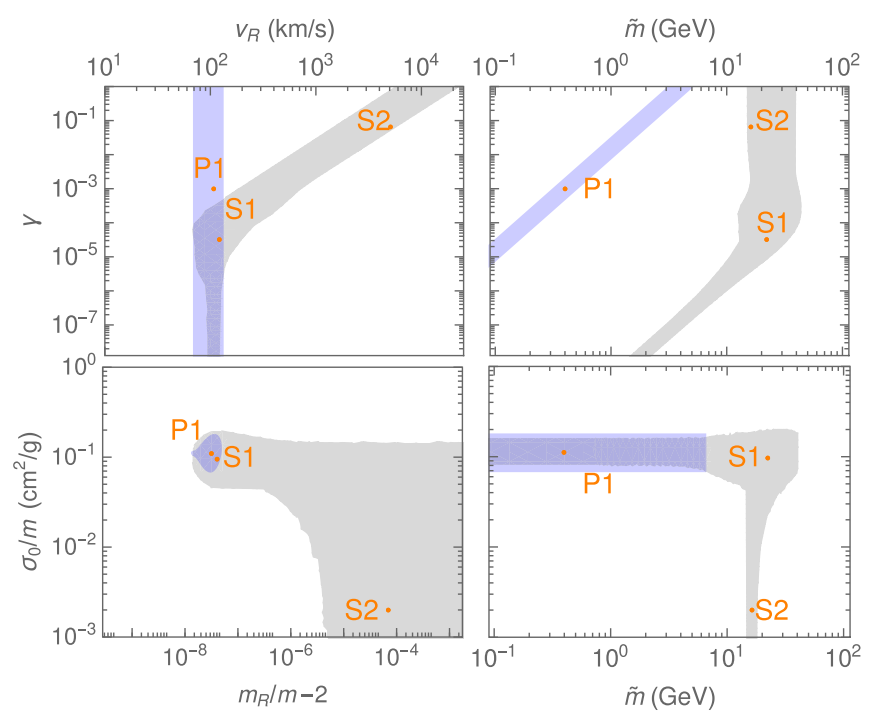

FIG. 2. $95 \%$ C.L. contours for $S$-wave (gray) and $P$-wave (purple) scatterings together with the corresponding parameter sets in Fig. 1. Notice that $m_{R} / m-2=v_{R}^{2} / 4$.

$$
\frac{1}{\left(v^{2}-v_{R}^{2}\right)^{2}+16 \gamma^{2} v^{2(2 L+1)}} \rightarrow \frac{\pi \delta\left(v-v_{R}\right)}{8 \gamma v_{R}^{2(L+1)}} .
$$

It works very well for $L \geq 1$, because $\gamma^{2} v^{2(2 L+1)} \ll v^{4}$. In this case, we find that $\mathcal{I}_{L}\left(\gamma, v_{R}, v_{0}\right)$ scales as $\gamma^{2} v_{0}^{4 L+1} / v_{R}^{4}$ at $v_{0} \ll v_{R}$ and as $\gamma^{2} v_{0}^{4 L-3}$ at $v_{0} \gg v_{R}$. In both regions, $\mathcal{I}_{L}$ cannot be much larger than one. Therefore, the resonant effect is negligible except for the intermediate region, where the NWA captures the velocity dependence as

$$
\left.\frac{\langle\sigma v\rangle}{m}\right|_{\mathrm{NWA}}=\frac{\sigma_{0}\langle v\rangle}{m}+\frac{128 S \pi^{3 / 2} \gamma v_{R}^{2 L+1}}{m^{3} v_{0}^{3}} e^{-v_{R}^{2} / v_{0}^{2}} .
$$

Notice that the peak lies at $v_{0} \sim v_{R}$ as illustrated by $P 1$ in Fig. 1. The corresponding line actually applies to any $L \geq 1$, because the dependence on $L$ can be absorbed by rescaling $m$. Using Eq. (7), we find that the best-fit parameters at 95\% C.L. for $L \geq 1$ are given by

$v_{R}=\left[108_{-43}^{+28}\right] \mathrm{km} / \mathrm{s}, \quad \sigma_{0} / m=\left[0.11_{-0.05}^{+0.10}\right] \mathrm{cm}^{2} / \mathrm{g}$,

$\tilde{m}=\left[400_{-90}^{+120}\right] \operatorname{MeV}\left(\frac{\gamma}{10^{-3}}\right)^{1 / 3}\left(\frac{v_{R}}{\left[3 \times 10^{5}\right] \mathrm{km} / \mathrm{s}}\right)^{2(L-1) / 3}$.

Such values for the velocity correspond to $m_{R} / m-2-10^{-7}$. The regions where all this applies are shown in Fig. 2. For $P$-wave scattering, demanding $\gamma \lesssim 1$ leads to $\tilde{m} \equiv m S^{-1 / 3} \lesssim 5 \mathrm{GeV}$. Moreover, a perturbative $\sigma_{0} / m$ around $0.1 \mathrm{~cm}^{2} / \mathrm{g}$ requires sub-GeV DM masses unless $S \gg 1$. Interestingly, $P 1$ predicts $\sigma / m \sim 0.1 \mathrm{~cm}^{2} / \mathrm{g}$ at $\langle v\rangle \ll 100 \mathrm{~km} / \mathrm{s}$. In fact, scatterings with $L \geq 1$ can realize small cross sections at very low velocities. Hence, the recent claim based on Draco observations [28] is consistent with RSIDM.

As long as $v_{R} \gtrsim 4 \gamma$, the NWA also applies for $S$-wave scattering. For $v_{R} \ll 4 \gamma, \mathcal{I}_{L}$ is proportional to $v_{0}$ (to $1 / v_{0}$ ) below (above) $v_{\text {peak }} \sim v_{R}^{2} /(4 \gamma) \ll v_{R}$, because such large values of $\gamma$ broaden the resonance. $S 1$ and $S 2$ illustrate the narrow and the broad width cases, respectively.

In conclusion, resonant scattering is able to address the observed DM mass deficit at all astrophysical scales.

RSIDM models.-Below, we illustrate the previous model-independent results in concrete RSIDM scenarios. We first introduce a Lagrangian specifying the coupling of the DM to the resonance (see Table I) and calculate the cross section and the self-energy. We subsequently corroborate that they can be cast as Eqs. (2) and (4) show. The scenarios are as follows.

(I) Fermionic DM with a pseudoscalar mediator.-The scattering process is $S$-wave while $\sigma_{0} \simeq 0$. The corresponding best fit is thus $S 2$. Notice that a light pseudoscalar mediator does not lead to SIDM, because it induces a 
TABLE I. Benchmark RSIDM models.

\begin{tabular}{lcccccc}
\hline \hline Scenario & Interaction Lagrangian & $L$ & $J_{\mathrm{DM}}$ & $J_{R}^{P}$ & $S$ & $\gamma$ \\
\hline $\mathrm{I}$ & $g R \overline{\mathrm{DM}} \gamma^{5} \mathrm{DM}$ & 0 & $\frac{1}{2}$ & $0^{-}$ & $\frac{1}{4}$ & $g^{2} / 32 \pi$ \\
$\mathrm{IIa}$ & $g R \mathrm{DM}^{i} \mathrm{DM}^{i}$ & 0 & 0 & $0^{+}$ & $\frac{1}{3}$ & $g^{2} / 16 \pi m_{R}^{2}$ \\
$\mathrm{IIb}$ & $g \epsilon_{i j k} R_{\mu}^{i} \mathrm{DM}^{j} \partial^{\mu} \mathrm{DM}^{k}$ & 1 & 0 & $1^{-}$ & 1 & $g^{2} / 384 \pi$ \\
$\mathrm{III}$ & $(1 / \Lambda) R_{\mu \nu} \mathcal{T}_{\mathrm{DM}}^{\mu \nu}$ & 2 & 0 & $2^{+}$ & 5 & $m_{R}^{2} / 30720 \pi \Lambda^{2}$ \\
\hline \hline
\end{tabular}

suppressed Yukawa potential (see, e.g., [85]). Because of this and because it leads to velocity-suppressed directdetection rates, this candidate is phenomenologically interesting.

(II) Dark mesons.-In QCD-like theories, DM can be a dark pion. Analogous to real pions, it can be a triplet $\mathrm{DM}^{i}$, with $i=1,2,3$. If $R$ is a dark $\sigma$ resonance (IIa), the scattering takes place via the $S$ wave, where we expect $\mathrm{GeV}$ $\mathrm{DM}$ and $\sigma_{0} / m \ll 100 \mathrm{~cm}^{2} / \mathrm{g}$. The best fit is thus $S 2$. If $R$ is a dark $\rho$ resonance (IIb), the scattering is $P$-wave suppressed. The constant piece of the cross section is given by $\sigma_{0} \sim \pi \gamma^{2} / \mathrm{m}^{2}$ in the perturbation theory, but it is plausible that there are other contributions. We therefore leave $\sigma_{0}$ as a free parameter. The corresponding best-fit curve is $P 1$. We expect $m \sim 400 \mathrm{MeV}$ in this case. In the same fashion, minimal QCD-like theories can also lead to spin-1 DM [86]. In all cases, DM can be produced by means of the strongly interacting massive particle (SIMP) [87-109] and the freeze-in [110-112] mechanisms.

(III) Tensor resonances.-They also arise in strongly coupled theories. Despite the potential complications of such theories, the generality of our approach allows us to describe the scattering induced by a spin- 2 resonance $R_{\mu \nu}$ [113]. If this couples to the DM energy-momentum tensor with a cutoff scale $\Lambda$, and taking scalar DM as an example, we find that the corresponding Feynman rules [114] indeed lead to a $D$-wave cross section given by Eq. (2). For $m \sim 10^{-3} \Lambda$, we obtain $\mathrm{keV} \mathrm{DM}$ with $\gamma \sim 10^{-13}$. The corresponding best fit is given by $P 1$ in Fig. 1 after rescaling the mass by means of Eq. (9).

Annihilation vs scattering.- It is not necessary that the DM annihilates, as, e.g., in models of asymmetric DM. Nonetheless, if the resonance decays into a pair of standard model (SM) particles $f \bar{f}$, in analogy to Eq. (2), the resonant $\mathrm{DM}$ annihilation into $f \bar{f}$ has a cross section

$$
\sigma_{\mathrm{anni}} \simeq \frac{4 \pi S}{m E(v)} \frac{\Gamma(v) m_{R} \gamma_{f} / 4}{\left[E(v)-E\left(v_{R}\right)\right]^{2}+\Gamma(v)^{2} / 4},
$$

where $m_{R} \gamma_{f}$ is the decay width for $R \rightarrow f \bar{f}$. As above, we assume that the resonance dominantly decays to a pair of DM particles and, thus, that the contribution of $f$ to the imaginary part of the resonance self-energy, $m_{R}^{2} \gamma_{f}$, is subleading. This is different from Ref. [70], in which the resonance dominantly decays into visible particles.

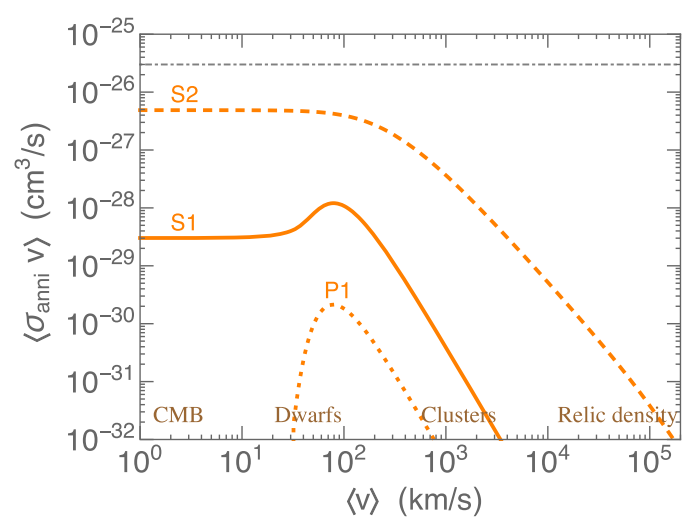

FIG. 3. Annihilation cross section into a pair of charged fermions for the parameter sets in Fig. 1, assuming a branching ratio $\gamma_{f} /\left(\gamma v_{R}^{2 L+1}\right)=10^{-13}$. The horizontal line gives the standard freeze-out benchmark.

As expected for annihilations (but not for elastic scatterings), $\sigma_{\text {anni }} v \propto v^{2 L}$ as long as $v \ll v_{R}$. Furthermore, for the cases where NWA applies, $\left.\left\langle\sigma_{\text {anni }} v\right\rangle\right|_{\text {peak }} \sim 32 \pi^{2} S \gamma_{f} /\left(m^{2} v_{R}^{3}\right)$. In contrast, for broad $S$-wave resonances such as $S 2$, where $v_{\text {peak }} \ll v_{R},\left.\left\langle\sigma_{\text {anni }} v\right\rangle\right|_{\text {peak }}$ gets enhanced by another factor $\left(v_{R} / v_{\text {peak }}\right)^{2 L+1}$.

The coupling to light charged particles is mostly constrained by Fermi-LAT observations of local satellites $[115,116]$ and the Planck data on the cosmic microwave background (CMB)[117,118]. For instance, the corresponding Fermi-LAT upper limit on $\left\langle\sigma v_{\text {anni }}\right\rangle$ for $\mathrm{GeV}$ DM is of the order of $10^{-26} \mathrm{~cm}^{3} / \mathrm{s}$. For $S 2$, this leads to an upper limit on the branching ratio, $\gamma_{f} /\left(\gamma v_{R}\right)$, of about $10^{-13}-10^{-12}$. This bound is much stronger than that of $S 1$ and $P 1$, due to the enhancement factor mentioned above. Motivated by this, we conservatively fix $\gamma_{f} /\left(\gamma v_{R}^{2 L+1}\right)=$ $10^{-13}$ and calculate the annihilation cross section as a function of $\langle v\rangle$ for the same parameter sets in Fig. 1. The result is shown in Fig. 3. Therefore, the resonance can couple only feebly to light charged particles, which is why the SIDM candidates with thermal freeze-out from Ref. [72] are excluded. Of course, this is model dependent. For instance, if the resonance couples only to neutrinos, the bound on $\left\langle\sigma_{\text {anni }} v\right\rangle$ becomes much weaker, and larger $\gamma_{f} / \gamma$ are thus allowed.

Furthermore, the strong velocity dependence of $\left\langle\sigma v_{\text {anni }}\right\rangle$ suggests that the usual freeze-out can hardly work, as for SIDM with light mediators decaying into visible particles [119-122]. Nevertheless, the DM abundance might arise from other SIDM production mechanisms [112]. Indeed, for the $S$-wave case, producing the DM abundance with small couplings is possible via freeze-in $[110,111]$ or 4-to-2 annihilations [97], where a scalar (vector) resonance can feebly mix with the Higgs (SM gauge bosons). See $[123,124]$ for reviews.

Discussion.-We advocate the resonant scattering as a possible SIDM realization with a velocity-dependent 
scattering cross section. Instead of a light mediator, this RSIDM scenario requires a near-threshold resonance with $m_{R} / m-2$ ranging from $\sim 10^{-7}$ for narrow resonances to $10^{-2}$ for $S$-wave scattering with broad widths. Such resonances exist in nature. As an example, $\alpha$ particles resonantly scatter by means of ${ }_{4}^{8} \mathrm{Be}$ in exactly the same way as described above. In fact, these processes were the main subject of the original article by Breit and Wigner [83], and they may as well occur in the DM sector. Actually, dark nucleons as SIDM have been studied in Refs. [73,125]. Furthermore, lattice studies suggest that QCD-like theories of DM might possess such states [126].

Conclusions. - We find that this RSIDM hypothesis can certainly address the core-vs-cusp and the too-to-big-fail problems while still being in agreement with cluster observations. We have also discussed indirect detection signatures, which are nevertheless model dependent. Additionally, we would like to emphasize that usual SIMPs - which are often said to be disfavored because their scattering cross section does not vary with velocitycan easily accommodate the mechanism proposed here.

We thank Ranjan Laha and Kai Schmidt-Hoberg for interesting discussions. X. C. is supported by the "New Frontiers" program of the Austrian Academy of Sciences. C. G.-C. is supported by the ERC Starting Grant NewAve (638528). H. M. thanks the Alexander von Humboldt Foundation for support while this work was completed. H. M. was supported by NSF Grant No. PHY-1638509, by U.S. DOE Contract No. DE-AC02-05CH11231, by the JSPS Grant-in-Aid for Scientific Research (C) (17K05409), by MEXT Grant-in-Aid for Scientific Research on Innovative Areas (15H05887, 15K21733), by WPI, MEXT, Japan, and by the Binational Science Foundation (Grant No. 2016153).

xiaoyong.chu@oeaw.ac.at †camilo.garcia.cely@desy.de *hitoshi@berkeley.edu, hitoshi.murayama@ipmu.jp

[1] J. Dubinski and R. G. Carlberg, The structure of cold dark matter halos, Astrophys. J. 378, 496 (1991).

[2] J. F. Navarro, C. S. Frenk, and S.D. M. White, The structure of cold dark matter halos, Astrophys. J. 462, 563 (1996).

[3] J. F. Navarro, C. S. Frenk, and S. D. M. White, A universal density profile from hierarchical clustering, Astrophys. J. 490, 493 (1997).

[4] B. Moore, Evidence against dissipationless dark matter from observations of galaxy haloes, Nature (London) 370, 629 (1994).

[5] R. A. Flores and J. R. Primack, Observational and theoretical constraints on singular dark matter halos, Astrophys. J. 427, L1 (1994).

[6] M. G. Walker and J. Penarrubia, A method for measuring (slopes of) the mass profiles of dwarf spheroidal galaxies, Astrophys. J. 742, 20 (2011).
[7] W. J. G. de Blok, S. S. McGaugh, A. Bosma, and V.C. Rubin, Mass density profiles of LSB galaxies, Astrophys. J. 552, L23 (2001).

[8] W. J. G. de Blok and A. Bosma, High-resolution rotation curves of low surface brightness galaxies, Astron. Astrophys. 385, 816 (2002).

[9] J. D. Simon, A. D. Bolatto, A. Leroy, L. Blitz, and E. L. Gates, High-resolution measurements of the halos of four dark matter-dominated galaxies: Deviations from a universal density profile, Astrophys. J. 621, 757 (2005).

[10] D. J. Sand, T. Treu, G. P. Smith, and R. S. Ellis, The dark matter distribution in the central regions of galaxy clusters: Implications for CDM, Astrophys. J. 604, 88 (2004).

[11] A. B. Newman, T. Treu, R. S. Ellis, and D. J. Sand, The density profiles of massive, relaxed galaxy clusters: II. Separating luminous and dark matter in cluster cores, Astrophys. J. 765, 25 (2013).

[12] M. Boylan-Kolchin, J. S. Bullock, and M. Kaplinghat, Too big to fail? The puzzling darkness of massive Milky Way subhaloes, Mon. Not. R. Astron. Soc. 415, L40 (2011).

[13] E. J. Tollerud, M. Boylan-Kolchin, and J. S. Bullock, M31 satellite masses compared to LCDM subhaloes, Mon. Not. R. Astron. Soc. 440, 3511 (2014).

[14] E. N. Kirby, J. S. Bullock, M. Boylan-Kolchin, M. Kaplinghat, and J. G. Cohen, The dynamics of isolated local group galaxies, Mon. Not. R. Astron. Soc. 439, 1015 (2014).

[15] W. J. G. d. Blok, A. Bosma, and S. S. McGaugh, Simulating observations of dark matter dominated galaxies: Towards the optimal halo profile, Mon. Not. R. Astron. Soc. 340, 657 (2003).

[16] G. Rhee, A. Klypin, and O. Valenzuela, The rotation curves of dwarf galaxies: A problem for cold dark matter?, Astrophys. J. 617, 1059 (2004).

[17] G. Gentile, A. Burkert, P. Salucci, U. Klein, and F. Walter, The dwarf galaxy DDO 47 as a dark matter laboratory: Testing cusps hiding in triaxial halos, Astrophys. J. Lett. 634, L145 (2005).

[18] K. Spekkens and R. Giovanelli, The cusp/core problem in galactic halos: Long-slit spectra for a large dwarf galaxy sample, Astron. J. 129, 2119 (2005).

[19] O. Valenzuela, G. Rhee, A. Klypin, F. Governato, G. Stinson, T. R. Quinn, and J. Wadsley, Is there evidence for flat cores in the halos of dwarf galaxies?: The case of ngc 3109 and ngc 6822, Astrophys. J. 657, 773 (2007).

[20] J. J. Dalcanton and A. Stilp, Pressure support in galaxy disks: Impact on rotation curves and dark matter density profiles, Astrophys. J. 721, 547 (2010).

[21] J. Kormendy and K. C. Freeman, Scaling laws for dark matter halos in late-type and dwarf spheroidal galaxies, Astrophys. J. 817, 84 (2016).

[22] J. I. Read, G. Iorio, O. Agertz, and F. Fraternali, Understanding the shape and diversity of dwarf galaxy rotation curves in $\Lambda$ CDM, Mon. Not. R. Astron. Soc. 462, 3628 (2016).

[23] A. V. Macciò, S. M. Udrescu, A. A. Dutton, A. Obreja, L. Wang, G. R. Stinson, and X. Kang, NIHAO X: Reconciling the local galaxy velocity function with cold dark matter via mock HI observations, Mon. Not. R. Astron. Soc. 463, L69 (2016). 
[24] E. Papastergis and A. A. Ponomareva, Testing core creation in hydrodynamical simulations using the HI kinematics of field dwarfs, Astron. Astrophys. 601, A1 (2017).

[25] A. M. Brooks, E. Papastergis, C. R. Christensen, F. Governato, A. Stilp, T. R. Quinn, and J. Wadsley, How to reconcile the observed velocity function of galaxies with theory, Astrophys. J. 850, 97 (2017).

[26] K. A. Oman, A. Marasco, J. F. Navarro, C. S. Frenk, J. Schaye, and A. Bentez-Llambay, Apparent cores and noncircular motions in the HI discs of simulated galaxies, Mon. Not. R. Astron. Soc. 482, 821 (2019).

[27] A. Genina, A. Benítez-Llambay, C. S. Frenk, S. Cole, A. Fattahi, J. F. Navarro, K. A. Oman, T. Sawala, and T. Theuns, The core-cusp problem: A matter of perspective, Mon. Not. R. Astron. Soc. 474, 1398 (2018).

[28] J. I. Read, M. G. Walker, and P. Steger, The case for a cold dark matter cusp in Draco, Mon. Not. R. Astron. Soc. 481, 860 (2018).

[29] J. F. Navarro, V. R. Eke, and C. S. Frenk, The cores of dwarf galaxy halos, Mon. Not. R. Astron. Soc. 283, L72 (1996).

[30] S. Gelato and J. Sommer-Larsen, On ddo154 and cold dark matter halo profiles, Mon. Not. R. Astron. Soc. 303, 321 (1999).

[31] J. Binney, O. Gerhard, and J. Silk, The dark matter problem in disk galaxies, Mon. Not. R. Astron. Soc. 321, 471 (2001).

[32] O. Y. Gnedin and H. Zhao, Maximum feedback and dark matter profiles of dwarf galaxies, Mon. Not. R. Astron. Soc. 333, 299 (2002).

[33] J. I. Read, M. G. Walker, and P. Steger, Dark matter heats up in dwarf galaxies, Mon. Not. R. Astron. Soc. 484, 1401 (2019).

[34] A. El-Zant, I. Shlosman, and Y. Hoffman, Dark halos: The flattening of the density cusp by dynamical friction, Astrophys. J. 560, 636 (2001).

[35] M. D. Weinberg and N. Katz, Bar-driven dark halo evolution: A resolution of the cusp-core controversy, Astrophys. J. 580, 627 (2002).

[36] K.-J. Ahn and P. R. Shapiro, Formation and evolution of the self-interacting dark matter halos, Mon. Not. R. Astron. Soc. 363, 1092 (2005).

[37] C. Tonini and A. Lapi, Angular momentum transfer in dark matter halos: Erasing the cusp, Astrophys. J. 649, 591 (2006).

[38] D. Martizzi, R. Teyssier, B. Moore, and T. Wentz, The effects of baryon physics, black holes and AGN feedback on the mass distribution in clusters of galaxies, Mon. Not. R. Astron. Soc. 422, 3081 (2012).

[39] D. N. Spergel and P. J. Steinhardt, Observational Evidence for Self-Interacting Cold Dark Matter, Phys. Rev. Lett. 84, 3760 (2000).

[40] R. Dave, D. N. Spergel, P. J. Steinhardt, and B. D. Wandelt, Halo properties in cosmological simulations of selfinteracting cold dark matter, Astrophys. J. 547, 574 (2001).

[41] M. Vogelsberger, J. Zavala, and A. Loeb, Subhaloes in self-interacting galactic dark matter haloes, Mon. Not. R. Astron. Soc. 423, 3740 (2012).

[42] M. Rocha, A. H. G. Peter, J. S. Bullock, M. Kaplinghat, S. Garrison-Kimmel, J. Onorbe, and L. A. Moustakas,
Cosmological simulations with self-interacting dark matter I: Constant density cores and substructure, Mon. Not. R. Astron. Soc. 430, 81 (2013).

[43] A. H. G. Peter, M. Rocha, J. S. Bullock, and M. Kaplinghat, Cosmological simulations with self-interacting dark matter II: Halo shapes vs. observations, Mon. Not. R. Astron. Soc. 430, 105 (2013).

[44] O. D. Elbert, J. S. Bullock, S. Garrison-Kimmel, M. Rocha, J. Oorbe, and A.H. G. Peter, Core formation in dwarf haloes with self-interacting dark matter: No finetuning necessary, Mon. Not. R. Astron. Soc. 453, 29 (2015).

[45] A. B. Fry, F. Governato, A. Pontzen, T. Quinn, M. Tremmel, L. Anderson, H. Menon, A. M. Brooks, and J. Wadsley, All about baryons: Revisiting SIDM predictions at small halo masses, Mon. Not. R. Astron. Soc. 452, 1468 (2015).

[46] Besides, SIDM can also explain the diversity of galaxy rotation curves [47-50].

[47] K. A. Oman et al., The unexpected diversity of dwarf galaxy rotation curves, Mon. Not. R. Astron. Soc. 452, 3650 (2015)

[48] A. Kamada, M. Kaplinghat, A. B. Pace, and H.-B. Yu, How the Self-Interacting Dark Matter Model Explains the Diverse Galactic Rotation Curves, Phys. Rev. Lett. 119, 111102 (2017).

[49] P. Creasey, O. Sameie, L. V. Sales, H.-B. Yu, M. Vogelsberger, and J. Zavala, Spreading out and staying sharp creating diverse rotation curves via baryonic and self-interaction effects, Mon. Not. R. Astron. Soc. 468, 2283 (2017).

[50] A. Robertson et al., The diverse density profiles of galaxy clusters with self-interacting dark matter plus baryons, Mon. Not. R. Astron. Soc. 476, L20 (2018).

[51] S. Tulin and H.-B. Yu, Dark matter self-interactions and small scale structure, Phys. Rep. 730, 1 (2018).

[52] M. Kaplinghat, S. Tulin, and H.-B. Yu, Dark Matter Halos as Particle Colliders: Unified Solution to Small-Scale Structure Puzzles from Dwarfs to Clusters, Phys. Rev. Lett. 116, 041302 (2016).

[53] J. L. Feng, M. Kaplinghat, and H.-B. Yu, Halo Shape and Relic Density Exclusions of Sommerfeld-Enhanced Dark Matter Explanations of Cosmic Ray Excesses, Phys. Rev. Lett. 104, 151301 (2010).

[54] S. D. McDermott, Is Self-Interacting Dark Matter Undergoing Dark Fusion?, Phys. Rev. Lett. 120, 221806 (2018).

[55] M. Vogelsberger, J. Zavala, K. Schutz, and T. R. Slatyer, Evaporating the Milky Way halo and its satellites with inelastic self-interacting dark matter, arXiv:1805.03203.

[56] A. Kamada, H. J. Kim, H. Kim, and T. Sekiguchi, SelfHeating Dark Matter via Semiannihilation, Phys. Rev. Lett. 120, 131802 (2018).

[57] X. Chu and C. Garcia-Cely, Core formation from selfheating dark matter, J. Cosmol. Astropart. Phys. 07 (2018) 013.

[58] A. Kamada, H. J. Kim, and H. Kim, Self-heating of strongly interacting massive particles, Phys. Rev. D 98, 023509 (2018).

[59] K. Griest and D. Seckel, Three exceptions in the calculation of relic abundances, Phys. Rev. D 43, 3191 (1991). 
[60] P. Gondolo and G. Gelmini, Cosmic abundances of stable particles: Improved analysis, Nucl. Phys. B360, 145 (1991).

[61] G. Jungman, M. Kamionkowski, and K. Griest, Supersymmetric dark matter, Phys. Rep. 267, 195 (1996).

[62] D. Feldman, Z. Liu, and P. Nath, PAMELA positron excess as a signal from the hidden sector, Phys. Rev. D 79, 063509 (2009).

[63] M. Pospelov and A. Ritz, Astrophysical signatures of secluded dark matter, Phys. Lett. B 671, 391 (2009).

[64] M. Ibe, H. Murayama, and T. T. Yanagida, Breit-Wigner enhancement of dark matter annihilation, Phys. Rev. D 79, 095009 (2009).

[65] J. D. March-Russell and S. M. West, WIMPonium and boost factors for indirect dark matter detection, Phys. Lett. B 676, 133 (2009).

[66] W.-L. Guo and Y.-L. Wu, Enhancement of dark matter annihilation via Breit-Wigner resonance, Phys. Rev. D 79, 055012 (2009).

[67] M. Ibe, Y. Nakayama, H. Murayama, and T. T. Yanagida, Nambu-Goldstone dark matter and cosmic ray electron and positron excess, J. High Energy Phys. 04 (2009) 087.

[68] M. Kakizaki, S. Matsumoto, Y. Sato, and M. Senami, Significant effects of second KK particles on LKP dark matter physics, Phys. Rev. D 71, 123522 (2005).

[69] C. Arina, T. Bringmann, J. Silk, and M. Vollmann, Enhanced line signals from annihilating Kaluza-Klein dark matter, Phys. Rev. D 90, 083506 (2014).

[70] M. Ibe and H.-b. Yu, Distinguishing dark matter annihilation enhancement scenarios via halo shapes, Phys. Lett. B 692, 70 (2010).

[71] E. Braaten and H. W. Hammer, Universal two-body physics in dark matter near an S-wave resonance, Phys. Rev. D 88, 063511 (2013).

[72] M. Duch and B. Grzadkowski, Resonance enhancement of dark matter interactions: The case for early kinetic decoupling and velocity dependent resonance width, J. High Energy Phys. 09 (2017) 159.

[73] E. Braaten, D. Kang, and R. Laha, Production of darkmatter bound states in the early universe by three-body recombination, J. High Energy Phys. 11 (2018) 084.

[74] For resonant scattering, $\sigma$ nearly equals the momentumtransfer cross section $\sigma_{T}$, and we do not differentiate between them here.

[75] R. Kuzio de Naray, S. S. McGaugh, and W. J. G. de Blok, Mass models for low surface brightness galaxies with high resolution optical velocity fields, Astrophys. J. 676, 920 (2008).

[76] S.-H. Oh, W. J. G. de Blok, E. Brinks, F. Walter, and R. C. Kennicutt, Jr., Dark and luminous matter in THINGS dwarf galaxies, Astron. J. 141, 193 (2011).

[77] O. D. Elbert, J. S. Bullock, M. Kaplinghat, S. GarrisonKimmel, A.S. Graus, and M. Rocha, A testable conspiracy: Simulating baryonic effects on self-interacting dark matter halos, Astrophys. J. 853, 109 (2018).

[78] M. Valli and H.-B. Yu, Dark matter self-interactions from the internal dynamics of dwarf spheroidals, Nat. Astron. 2, 907 (2018).

[79] A. Sokolenko, K. Bondarenko, T. Brinckmann, J. Zavala, M. Vogelsberger, T. Bringmann, and A. Boyarsky, Towards an improved model of self-interacting dark matter haloes, J. Cosmol. Astropart. Phys. 12 (2018) 038.

[80] S. W. Randall, M. Markevitch, D. Clowe, A. H. Gonzalez, and M. Bradac, Constraints on the self-interaction crosssection of dark matter from numerical simulations of the merging galaxy cluster 1E 0657-56, Astrophys. J. 679, 1173 (2008).

[81] A. Robertson, R. Massey, and V. Eke, What does the bullet cluster tell us about self-interacting dark matter?, Mon. Not. R. Astron. Soc. 465, 569 (2017).

[82] Note that the interference term exists only for $S$-wave scattering. For the cases discussed here, that term was found to be negligible with respect to the second term in Eq. (2). Furthermore, it changes its sign from below to above resonance and, hence, nearly cancels out upon integration over the velocity profile.

[83] G. Breit and E. Wigner, Capture of slow neutrons, Phys. Rev. 49, 519 (1936).

[84] Using a similar method, $\langle\sigma v\rangle / m$ has been estimated for eight Milky Way dwarfs [78]. A proper combined fit including those results is beyond the scope of this Letter. Nevertheless, since such dwarf galaxies all have approximately the same $\langle v\rangle$, a combined fit would not change our conclusions regarding the velocity dependence of $\langle\sigma v\rangle$.

[85] F. Kahlhoefer, K. Schmidt-Hoberg, and S. Wild, Dark matter self-interactions from a general spin-0 mediator, J. Cosmol. Astropart. Phys. 08 (2017) 003.

[86] A. Francis, R. J. Hudspith, R. Lewis, and S. Tulin, Dark Matter from strong dynamics: The minimal theory of dark baryons, J. High Energy Phys. 12 (2018) 118.

[87] A. D. Dolgov, On concentration of relict theta particles, Yad. Fiz. 31, 1522 (1980).

[88] E. D. Carlson, M. E. Machacek, and L. J. Hall, Selfinteracting dark matter, Astrophys. J. 398, 43 (1992).

[89] Y. Hochberg, E. Kuflik, T. Volansky, and J. G. Wacker, Mechanism for Thermal Relic Dark Matter of Strongly Interacting Massive Particles, Phys. Rev. Lett. 113, 171301 (2014).

[90] N. Yamanaka, S. Fujibayashi, S. Gongyo, and H. Iida, Dark matter in the hidden gauge theory, arXiv:1411 .2172 .

[91] Y. Hochberg, E. Kuflik, H. Murayama, T. Volansky, and J. G. Wacker, Model for Thermal Relic Dark Matter of Strongly Interacting Massive Particles, Phys. Rev. Lett. 115, 021301 (2015).

[92] N. Bernal, C. Garcia-Cely, and R. Rosenfeld, WIMP and SIMP dark matter from the spontaneous breaking of a global group, J. Cosmol. Astropart. Phys. 04 (2015) 012.

[93] N. Bernal, C. Garcia-Cely, and R. Rosenfeld, $\mathbb{Z}_{3}$ WIMP and SIMP dark matter from a global U(1) breaking, Nucl. Part. Phys. Proc. 267-269, 353 (2015).

[94] H. M. Lee and M.-S. Seo, Communication with SIMP dark mesons via Z' -portal, Phys. Lett. B 748, 316 (2015).

[95] S.-M. Choi and H. M. Lee, SIMP dark matter with gauged $\mathbb{Z}_{3}$ symmetry, J. High Energy Phys. 09 (2015) 063.

[96] M. Hansen, K. Langaeble, and F. Sannino, SIMP model at NNLO in chiral perturbation theory, Phys. Rev. D 92, 075036 (2015).

[97] N. Bernal and X. Chu, $\mathbb{Z}_{2}$ SIMP dark matter, J. Cosmol. Astropart. Phys. 01 (2016) 006. 
[98] E. Kuflik, M. Perelstein, N. R. -L. Lorier, and Y.-D. Tsai, Elastically Decoupling Dark Matter, Phys. Rev. Lett. 116, 221302 (2016).

[99] Y. Hochberg, E. Kuflik, and H. Murayama, SIMP Spectroscopy, J. High Energy Phys. 05 (2016) 090.

[100] S.-M. Choi and H. M. Lee, Resonant SIMP dark matter, Phys. Lett. B 758, 47 (2016).

[101] D. Pappadopulo, J. T. Ruderman, and G. Trevisan, Dark matter freeze-out in a nonrelativistic sector, Phys. Rev. D 94, 035005 (2016).

[102] M. Farina, D. Pappadopulo, J. T. Ruderman, and G. Trevisan, Phases of cannibal dark matter, J. High Energy Phys. 12 (2016) 039.

[103] S.-M. Choi, Y.-J. Kang, and H. M. Lee, On thermal production of self-interacting dark matter, J. High Energy Phys. 12 (2016) 099.

[104] U. K. Dey, T. N. Maity, and T. S. Ray, Light dark matter through assisted annihilation, J. Cosmol. Astropart. Phys. 03 (2017) 045.

[105] J. Cline, H. Liu, T. Slatyer, and W. Xue, Enabling forbidden dark matter, Phys. Rev. D 96, 083521 (2017).

[106] S.-M. Choi, H. M. Lee, and M.-S. Seo, Cosmic abundances of SIMP dark matter, J. High Energy Phys. 04 (2017) 154.

[107] S.-M. Choi, Y. Hochberg, E. Kuflik, H. M. Lee, Y. Mambrini, H. Murayama, and M. Pierre, Vector SIMP dark matter, J. High Energy Phys. 10 (2017) 162.

[108] X. Chu and C. Garcia-Cely, Self-interacting spin-2 dark matter, Phys. Rev. D 96, 103519 (2017).

[109] S.-M. Choi, H. M. Lee, P. Ko, and A. Natale, Resolving phenomenological problems with strongly-interactingmassive-particle models with dark vector resonances, Phys. Rev. D 98, 015034 (2018).

[110] J. McDonald, Thermally Generated Gauge Singlet Scalars as Self-Interacting Dark Matter, Phys. Rev. Lett. 88, 091304 (2002).

[111] L. J. Hall, K. Jedamzik, J. March-Russell, and S. M. West, Freeze-in production of FIMP dark matter, J. High Energy Phys. 03 (2010) 080.

[112] N. Bernal, X. Chu, C. Garcia-Cely, T. Hambye, and B. Zaldivar, Production regimes for self-interacting dark matter, J. Cosmol. Astropart. Phys. 03 (2016) 018.

[113] Not to be confused with the Ricci tensor.

[114] T. Han, J. D. Lykken, and R.-J. Zhang, On Kaluza-Klein states from large extra dimensions, Phys. Rev. D 59, 105006 (1999).
[115] M. Ackermann et al. (Fermi-LAT Collaboration), Dark matter constraints from observations of 25 Milky Way satellite galaxies with the Fermi Large Area Telescope, Phys. Rev. D 89, 042001 (2014).

[116] A. Albert et al. (DES and Fermi-LAT Collaborations), Searching for dark matter annihilation in recently discovered Milky Way satellites with Fermi-LAT, Astrophys. J. 834, 110 (2017).

[117] P. A. R. Ade et al. (Planck Collaboration), Planck 2015 results. XIII. Cosmological parameters, Astron. Astrophys. 594, A13 (2016).

[118] H. Liu, T. R. Slatyer, and J. Zavala, Contributions to cosmic reionization from dark matter annihilation and decay, Phys. Rev. D 94, 063507 (2016).

[119] T. Bringmann, F. Kahlhoefer, K. Schmidt-Hoberg, and P. Walia, Strong Constraints on Self-Interacting Dark Matter with Light Mediators, Phys. Rev. Lett. 118, 141802 (2017).

[120] T. Binder, M. Gustafsson, A. Kamada, S. M. R. Sandner, and M. Wiesner, Reannihilation of self-interacting dark matter, Phys. Rev. D 97, 123004 (2018).

[121] M. Hufnagel, K. Schmidt-Hoberg, and S. Wild, BBN constraints on MeV-scale dark sectors. Part I. Sterile decays, J. Cosmol. Astropart. Phys. 02 (2018) 044.

[122] M. Hufnagel, K. Schmidt-Hoberg, and S. Wild, BBN constraints on $\mathrm{MeV}$-scale dark sectors. Part II. Electromagnetic decays, J. Cosmol. Astropart. Phys. 11 (2018) 032.

[123] R. Essig et al., Working Group report: New light weakly coupled particles, in Proceedings of the 2013 Community Summer Study on the Future of U.S. Particle Physics: Snowmass on the Mississippi (CSS2013): Minneapolis, MN, 2013 (SLAC National Accelerator Laboratory, 2013), http://www.slac.stanford.edu/econf/C1307292/docs/ IntensityFrontier/NewLight-17.pdf.

[124] N. Bernal, M. Heikinheimo, T. Tenkanen, K. Tuominen, and V. Vaskonen, The dawn of FIMP dark matter: A review of models and constraints, Int. J. Mod. Phys. A 32, 1730023 (2017).

[125] Their findings suggest that their model is described by $S 2$.

[126] R. A. Briceno, J. J. Dudek, and R. D. Young, Scattering processes and resonances from lattice QCD, Rev. Mod. Phys. 90, 025001 (2018). 